\title{
Centrifugal spun ultrafine fibrous web as a potential drug delivery vehicle
}

\author{
L. Amalorpava Mary ${ }^{1}$, T. Senthilram ${ }^{1}$, S. Suganya ${ }^{1}$, L. Nagarajan ${ }^{1}$ J. Venugopal ${ }^{2}$, \\ S. Ramakrishna ${ }^{2}$, V. R. Giri Dev ${ }^{1 *}$ \\ ${ }^{1}$ Department of Textile Technology, Anna University, Chennai-25, India \\ ${ }^{2}$ Nanoscience and Nanotechnology Initiative, Faculty of Engineering, National University of Singapore, Singapore
}

Received 1 September 2012; accepted in revised form 10 November 2012

\begin{abstract}
Centrifugal spinning (C-spin) is one of the emerging techniques for the production of ultrafine fibrous web which mimics Extracellular matrix (ECM). Due to its unique characteristic features it is widely used in bio-medical applications such as tissue engineered scaffolds, wound dressing materials and drug delivery vehicles. In the present study tetracycline loaded polycaprolactone (PCL) blended polyvinyl pyrrolidone (PVP) fibers were fabricated using in-house built C-spin system. The developed ultrafine fibers were morphologically characterized by Scanning Electron Microscope (SEM) before and after drug release and the results showed that the developed webs were highly porous and the pores were evenly distributed. Fourier Transform Infrared (FTIR) spectroscopy results confirmed that the drug was incorporated on the fibers. The antibacterial activity and drug releasing strategy were examined and the results showed that the developed webs can effectively act as a drug delivery vehicle.
\end{abstract}

Keywords: nanomaterials, centrifugal spinning, drug delivery, wound dressing, PCL/PVP/Tetracycline scaffolds

\section{Introduction}

Over the last few decades, ultrafine fibers webs have engrossed the interest of the researchers for its innovative applications. Due to its captivating characteristics features such as large surface area-tovolume ratio, high porosity, and fully interconnected pore network it has been widely used in applications like drug delivery, wound dressings, tissue engineered scaffolds, protective clothing, filtration systems, sanitary goods, and sensor assemblies [1,2]. At present ultrafine fibers are produced by phase separation, template synthesis, self-assembly, electrospinning [3-5]. Among these, electrospinning is the most promising and versatile technique for the production of ultrafine fibers. But the utilization of electrospinning is limited by the major disadvantages like low production rate, low safety features and getting aligned fibers. Research attempts are being made to replace electrospinning with alternative techniques around the globe. Forced spinning and rotary jet spinning techniques are now being attempted to produce aligned fibers without application of high voltage. This facile method of centrifugal spinning can be able to replace the limitations of electro spinning and the process is highly scalable.

Centrifugal spinning system is developed based on cotton candy machine working principle. Polymers are drawn into fibers by applying high centrifugal force. Centrifugal spinning process offers several appealing features such as the obtained fibrous web shows high surface area to volume ratio (the diameter of the fibers varies from several micrometers down to nano meters range), webs with higher

\footnotetext{
${ }^{*}$ Corresponding author, e-mail: vrgiridev@yahoo.com

(C) BME-PT
} 
degree of alignment and interconnected fibers offers good mechanical strength, highly porous, well interconnected pores, facile and low cost effective process. Because of these cynosural characteristics of ultrafine fibers produced by this process can be used as a tissue engineering scaffolds (especially those needing aligned architecture such as nerve, muscle, tendons), drug delivery vehicles and wound dressing materials [6]. An ideal wound dressing material should possess moderately hydrophilic surface, dimensional un-shrinkage, suitable microstructure, porosity, controllable biodegradability, suitable mechanical property and should support initial cell adhesion and proliferation [7-9].

PCL is Food and Drug administration (FDA) approved semi crystalline aliphatic polyester having an excellent biocompatibility and degradability. When compared to other biocompatible polymers such as poly(lactic-co-glycolic acid) (PLGA), it does not create an acidic environment on degradation $[10,11]$. Even though it possesses an excellent processability and mechanical properties, utilization of PCL in wound dressings is limited because of its hydrophobicity. Hydrophobic polymers provide a structure or back bone and degrade over a long period whereas the more hydrophilic polymers degrade rapidly. The choice of polymer and polymer blends plays an important role where it is aimed as a vehicle for controlled release. PVP is one of the important hydrophilic polymers that can be blended homogeneously with PCL. Due to its good complexion, adhesion properties, excellent physiological compatibility, low chemical toxicity, and reasonable solubility it has been widely used in many industries such as pharmaceuticals, cosmetics, beverages, adhesives, detergents, paints, electronics, and biological engineering materials $[12,13]$.

Some trail works have been done with electrospun PCL nanofibrous web as a drug releasing vehicle. Metronidazole benzoate loaded PCL fibrous web was prepared for targeting periodontal disorder by electrospinning and drug releasing behaviors has been studied [14]. Controlled release of diclofenac sodium and teracyclin hydrochloride from electrospun PCL, polyvinyl alcohol, PVA-SA (Sodium alginate) has also been reported [15]. Release of tetracycline from electrospun mats of Poly (ethylene- co- vinyl acetate) (PEVA), PLA 50/50 blend was studied and was found that electrospun PEVA and 50/50 PLA/PEVA mats gave relatively smooth release of drug electrospun fibers after release over about 5 days [16].

To the best of our knowledge, no attempt has been made with centrifugal spun fibrous web as a drug carrier. Still no report has been found for centrifugal spinning of PCL/PVP blends. Hence, studying the release pattern of drug from the centrifugal spun mat will provide insight to the usage of material as the drug delivery vehicle. In the present study PCL/ PVP ultrafine fiber mats were prepared by in-house developed centrifugal spinning setup. Drug was loaded on the mat and its releasing profiles were studied, the efficiency of the mat against skin pathogens are also examined and discussed for wound healing.

\section{Experimental}

\subsection{Materials}

Polycaprolactone ( $\left.M_{\mathrm{n}} 70000-90000\right)$, polyvinyl pyrrolidone $\left(M_{\mathrm{w}} 1300 \mathrm{KD}\right)$ and tetracycline were obtained from Sigma Aldrich India. Chloroform and Methanol (RANKEM, India) used in this study were analytical grade and used without further purification.

\subsection{Preparation of ultrafine fibrous web by centrifugal spinning}

Centrifugal spinning was carried out using $12 \% \mathrm{w} / \mathrm{v}$ solutions of PCL/PVP in the ratio of 100:0, 70:30, 50:50, 30:70, 0:100 in chloroform. Drug loaded samples were prepared by dissolving $0.2 \% \mathrm{w} / \mathrm{v}$ of tetracycline with respect to polymer concentration in 10:90 methanol:chloroform mixture. Prior to centrifugal spinning the solutions were stirred for an hour. Both drug free and drug loaded samples were carefully injected into the pot type rotating spinneret head with the help of syringe. Centrifugal spinning was carefully carried out under a constant $2000 \mathrm{rpm}$. The formed ultrafine fibers were collected on a round bottom collector. The obtained aligned fibrous web was kept under vacuum to remove the excess solvent. Schematic diagram of the in-house developed centrifugal spinning system is given in Figure 1. The actual drug loaded in the samples was found out by the procedure cited in literature with chloroform as solvent and it was found be $96-99 \%$ of the initial loading of drug in the samples [17]. 


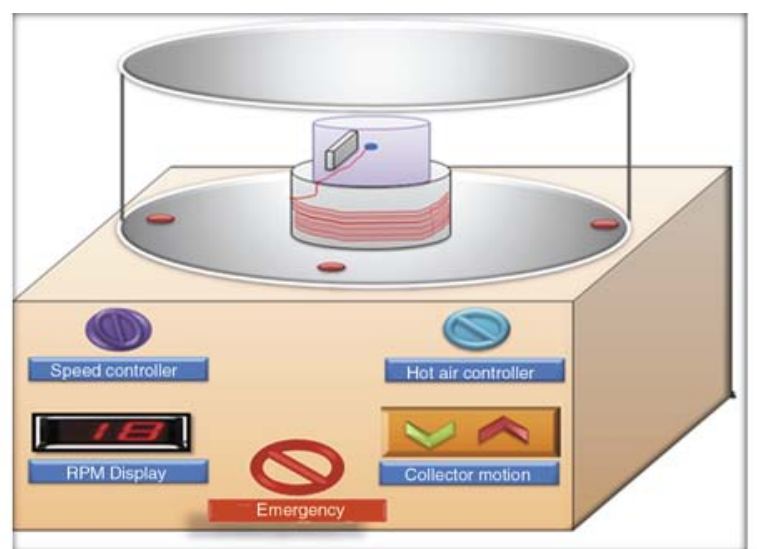

a)

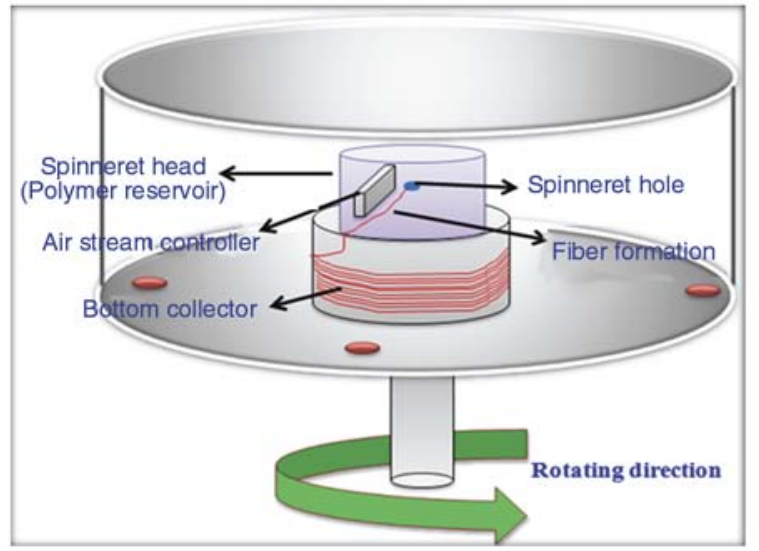

c)

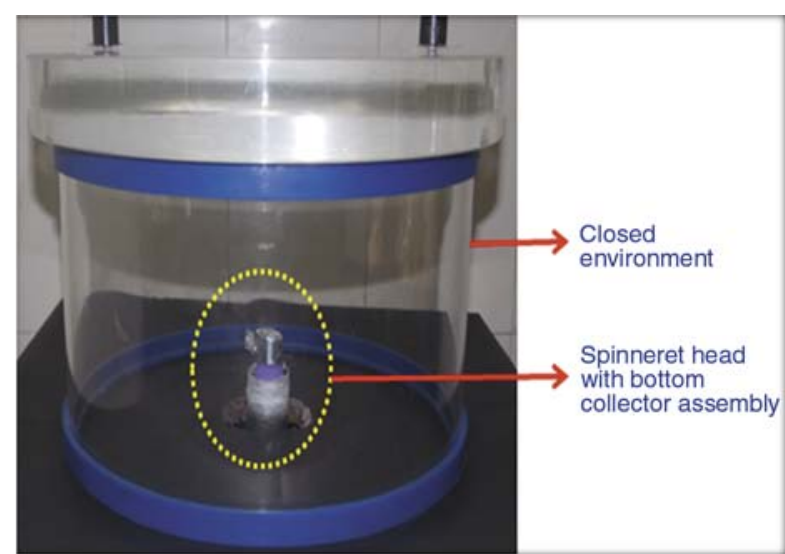

b)

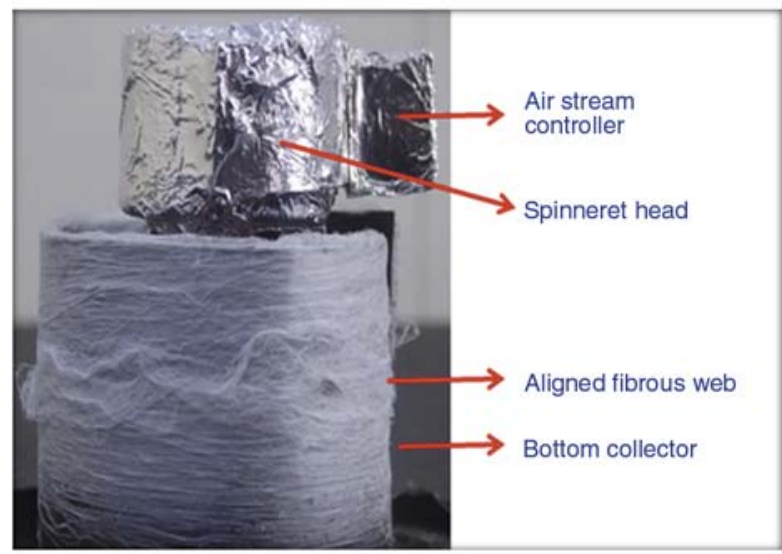

d)

Figure 1. Schematic diagram of the in-house developed centrifugal spinning system, (a) centrifugal spinning set up (b) fiber production (c) rotating pot type spinneret head (d) aligned fibers on bottom collector

\subsection{Characterization of drug loaded fibers}

The morphology of the ultrafine fibrous web was observed using scanning electron microscope (SEM) (S3400NSEM, HITACHI, Japan). The spun fibers were sputtered with thin layer of gold prior to SEM observation. On the basis of SEM images, the diameter of the fibers was calculated with the help of image analyzing software Digimizer. ATR-FTIR spectroscopic analysis of centrifugal spun ultrafine fibrous web was made using Spectrum One (PerkinElmer, USA model). The thermal behavior of centrifugal spun fibrous web samples (PCL, PCL/PVP, $\mathrm{PCL} / \mathrm{PVP} /$ Tetracycline and PVP) was examined by TA Q50 series Thermo Gravimetric Analyzer (TGA, USA). Measurements were conducted over a temperature range of 0 to $700^{\circ} \mathrm{C}$ at a heating rate of $20^{\circ} \mathrm{C} /$ min under nitrogen purge.

\subsection{Swelling and degradation studies}

The degree of swelling and weight loss of the drug loaded ultrafine fibrous mats were calculated by using Equations (1) and (2) respectively:
Degree of swelling [\%] $=\frac{M-M_{\mathrm{d}}}{M_{\mathrm{d}}} \cdot 100$

Weight loss [\%] $=\frac{M_{\mathrm{i}}-M_{\mathrm{d}}}{M_{\mathrm{i}}} \cdot 100$

where $M$ is weight of each sample submersion in the buffer solution which is wiped dry with filter paper, $M_{\mathrm{d}}$ is the dry weight of immersed sample which is dried at $40^{\circ} \mathrm{C}$ and $M_{\mathrm{i}}$ is the initial weight of the sample in its dry state [18]. Both tests were carried out in the acetate buffer of $\mathrm{pH} 5.5$ at $37^{\circ} \mathrm{C}$ for $2,4,12$ and 24 hours.

\subsection{Antimicrobial activity}

The antibacterial activity of the centrifugal spun fibrous web against four pathogenic bacteria commonly found on burn wounds: P.aeruginosa (Gramnegative) S.epidermidis (Gram-positive), B.magaterium (Gram-positive) and E.coli (Gram-negative) were investigated. The assessment was conducted based on the disc agar diffusion method. A $100 \mu \mathrm{L}$ 
aliquot of bacteria reconstituted in nutrient broth and previously subcultured was spread onto an agar plate. Both the drug-free and drug-containing PCL/ PVP fiber mats were cut into circular discs $(10 \mathrm{~mm}$ in diameter) and placed on the top of the agar plate. The plates were incubated at $37^{\circ} \mathrm{C}$ for $24 \mathrm{~h}$. If inhibitory concentrations were reached, there would be no growth of the microbes, which could be seen as a clear zone around the disc specimens. The zone was then recorded as an indication of inhibition against the microbial species.

\subsection{In vitro drug release studies}

A drug-containing fiber mat $(0.5 \mathrm{~g})$ was first placed in a vial filled with $20 \mathrm{ml}$ of release medium acetate buffer. Drug release studies were carried out at $37^{\circ} \mathrm{C}$ and 100 rotation/min [rpm] in a thermostated shaking incubator. The releasing medium acetate buffer with $\mathrm{pH} 5.5$ was prepared by dissolving $1.5 \mathrm{~g}$ of sodium acetate in $1.5 \mathrm{~mL}$ of glacial acetic acid and then the final solution was made up to $100 \mathrm{~mL}$ by adding distilled water. In this case, $4 \mathrm{~mL}$ of sample was taken from the medium after appropriate intervals for about $24 \mathrm{~h}$ and then the same volume of fresh release medium was added as replacement. A calibration curve was obtained for the tetracycline hydrochloride concentration at a peak absorption wavelength of $360 \mathrm{~nm}$, and a linear equation was derived by a curve-fitting method. In the assessment of drug release behavior, a cumulated amount of the released drug was calculated. The percentages of drug released from the fibrous web were plotted against time. The drug release studies were carried out on PCL and PCL/PVP blended fibrous mat alone and the kinetics studies were carried out on the drug release from the mat using the Zero order, First order, Higuchi, Hixson-crowell and Peppas model as suggested in the literature [19-23].

\section{Results and discussion}

\subsection{Scanning electron microscope analysis}

Wound care products are likely to be sophisticated formulations that incorporate antimicrobial components and optimize the wound environment to promote healing. Since evolution is faster in microbial species than in other species, there will be a continual need to search for novel wound dressing materials for tropical skin infection treatments. Morphologies of C-spun fibrous webs are given in the Figure 2. The average diameter of the fibers calculated using
Table 1. Measurements of average fibre diameter of C-spun ultrafine fibres

\begin{tabular}{|c|c|}
\hline $\begin{array}{c}\text { PCL/ PVP Blend ratio } \\
{[\%]}\end{array}$ & $\begin{array}{c}\text { Average fiber diameter } \\
{[\mathbf{n m}]}\end{array}$ \\
\hline $100 / 0$ & 311 \\
\hline $70 / 30$ & 352 \\
\hline $50 / 50$ & 619 \\
\hline $50 / 50 /$ Tetracycline & 927 \\
\hline $30 / 70$ & 726 \\
\hline $0 / 100$ & 823 \\
\hline
\end{tabular}

Digimizer software is given in Table 1. The diameter of the fibers produced from $\mathrm{C}$-spun fiber ranges from 300 to $927 \mathrm{~nm}$. It can be inferred from the table that the addition of PVP to PCL increases the diameter of the fiber. The increase in diameter of the fiber due to the addition of PVP can be attributed to the hydrophilic nature of PVP. It can absorb the moisture present in air and can coalesce with other fibers leading to increase in diameter. The addition of drug to PCL/PVP (50/50) blended samples further increases the diameter as the drug occupies matrices of the polymer structure. However the changes in diameter of the PCL fibers loaded with drugs were not substantial when compared to that of the blends. The surface of C-spun fibers are rough and lack luster where as with corporation of PVP, the samples look lustrous due to the presence of homogeneity in diameter of the fibers. The photograph of the sample is given in the Figure 3. Another interesting observation is that the obtained fibers are highly aligned and the fiber alignment is mainly due to the presence of air stream controller which is placed near to the fine orifice. When the polymers are ejected from the narrow orifice with high centrifugal force, the air stream controller push the fibers down wards and paving a path for collecting at the bottom with higher degree of alignment. It has been reported in literature that the high degree of alignment has a significant influence on the drug release behavior after the initial burst release due to enhanced density of packing of fibers and limited pore size in aligned mats. It has also been reported in the literature that alignment of fibers supplements the proliferation rate of fibroblasts cells apart from providing good mechanical properties [24, 25].

\subsection{Fourier transform infrared spectroscopic analysis}

The FTIR spectrum of PCL is shown in the Figure 4a. The spectrum showed characteristic peaks 


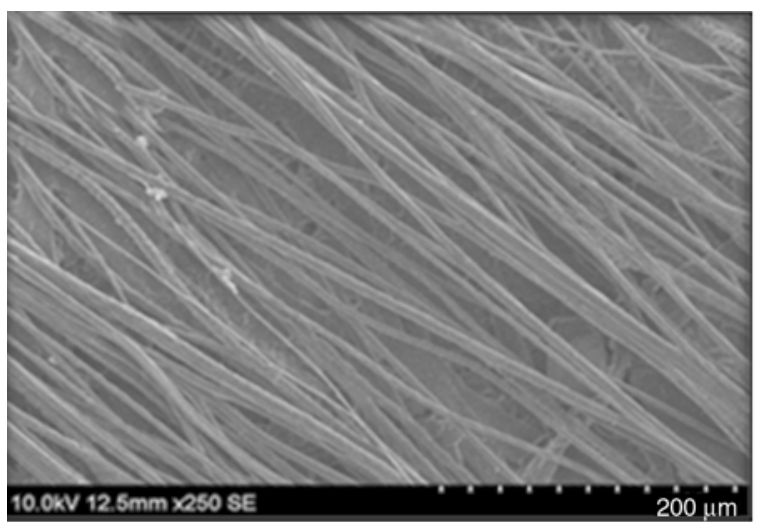

a)

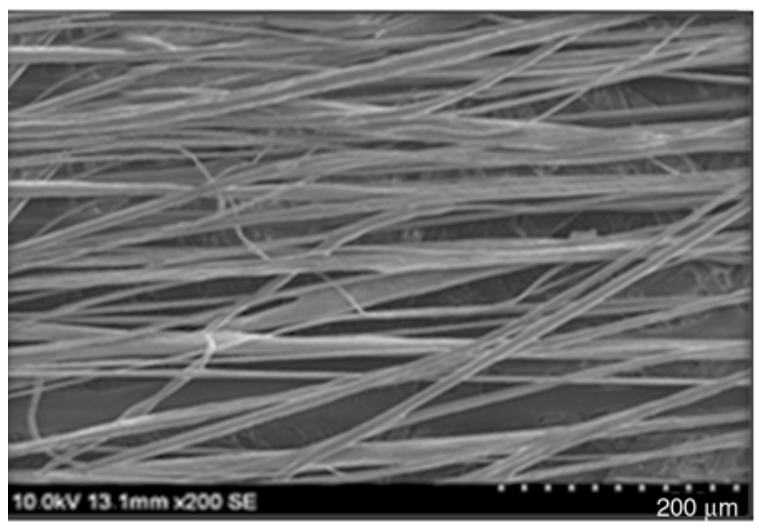

c)

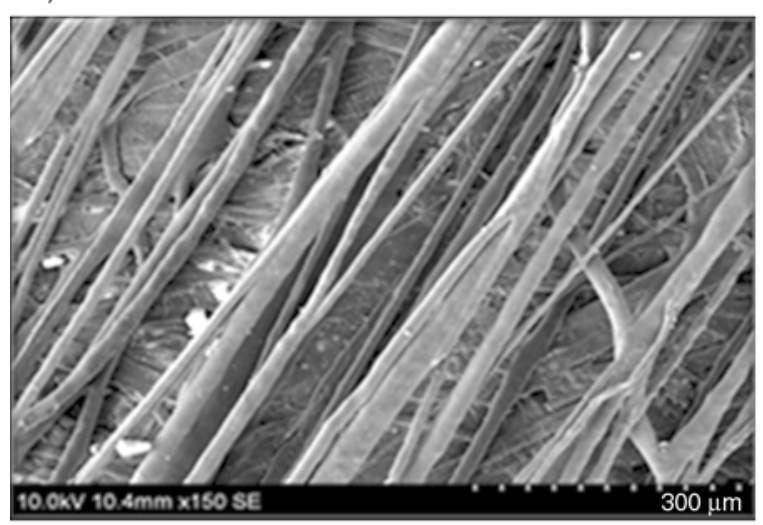

e)

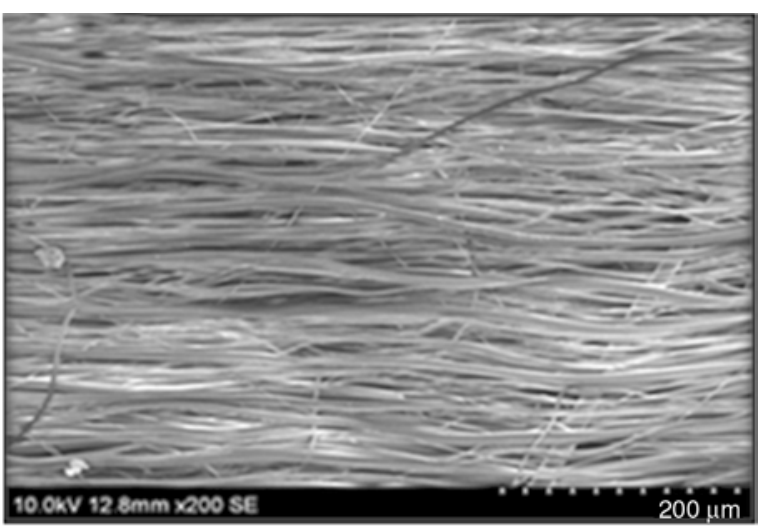

b)

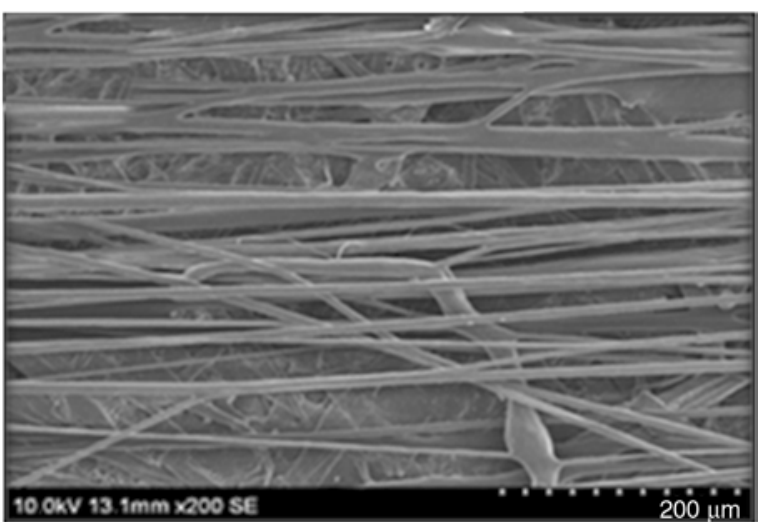

d)

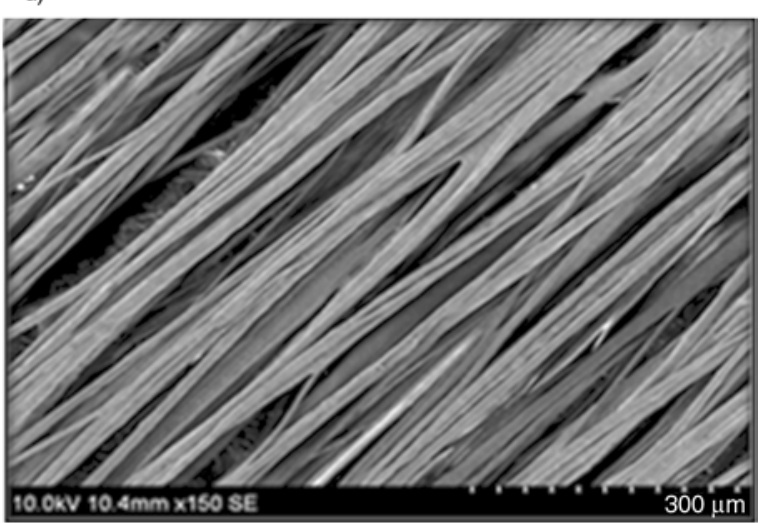

f)

Figure 2. SEM micrographs of (a) 100\% PCL (b) 100\% PVP (c) 70:30 PCL/PVP (d) 30:70 PCL/PVP (e) 50:50 PCL/PVP (f) Drug loaded PCL/PVP

of $\mathrm{C}=\mathrm{O}$ stretching vibrations at $1731 \mathrm{~cm}^{-1}, \mathrm{CH}_{2}$ bending modes at 1471, 1397 and $1365 \mathrm{~cm}^{-1}$ and -COO- vibrations at $1739 \mathrm{~cm}^{-1}$. The $\mathrm{C}-\mathrm{O}-\mathrm{C}$ stretching vibrations yield peaks at 1169, 1108 and $1049 \mathrm{~cm}^{-1}$. The spectrum of PVP is given in Figure $4 \mathrm{~b}$. The intense sharp peak at $1654 \mathrm{~cm}^{-1}$ appeared due to $\mathrm{C}=\mathrm{O}$ stretching vibrations of PVP. The $\mathrm{CH}_{2}$ bending modes were observed at 1376, 1424, 1441 and $1463 \mathrm{~cm}^{-1}$ and that of the $\mathrm{C}-\mathrm{N}$ vibrations occurred at $1291 \mathrm{~cm}^{-1}$. The spectrum of PCL/PVP $(50 / 50)$ shown in Figure 4c. The spectrum showed characteristics of both PCL $(\mathrm{C}=\mathrm{O}$ stretch at $\left.1734 \mathrm{~cm}^{-1}\right)$ and PVP $\left(\mathrm{C}=\mathrm{O}\right.$ stretch at $\left.1654 \mathrm{~cm}^{-1}\right)$. In addition to the above peaks, the $-\mathrm{COO}$ stretch of PCL occurred at $1239 \mathrm{~cm}^{-1}$ and C-N stretch of PVP at $1293 \mathrm{~cm}^{-1}$. The spectrum of drug loaded PCL/ PVP (50/50) is given in Figure 4d. The spectrum showed the characteristics peaks of PCL and PVP. But the $\mathrm{C}=\mathrm{O}$ stretching vibrations of $\mathrm{PCL}$ and $\mathrm{PVP}$ showed splits due to interactions of polymer with the drug. Further, the $\mathrm{NH}_{2}$ symmetric and asymmetric stretching vibrations at 3300 and $3415 \mathrm{~cm}^{-1}$ was partly resolved and it confirms the presence of drug. 


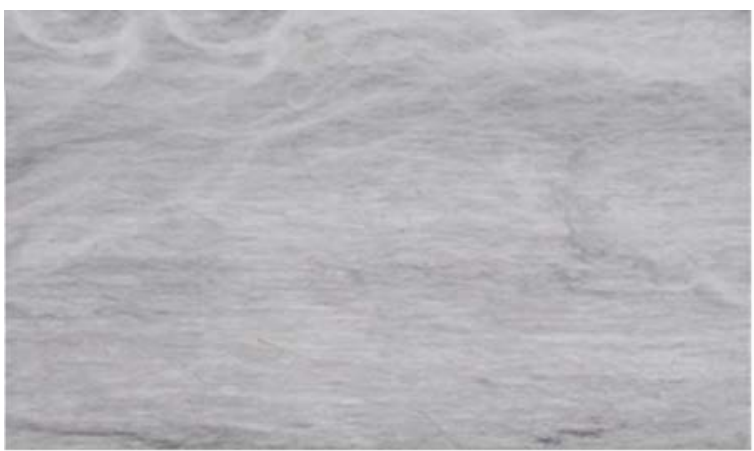

a)

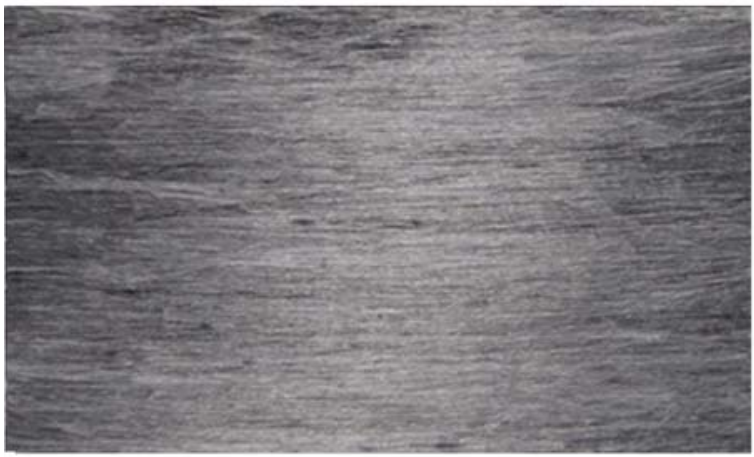

c)

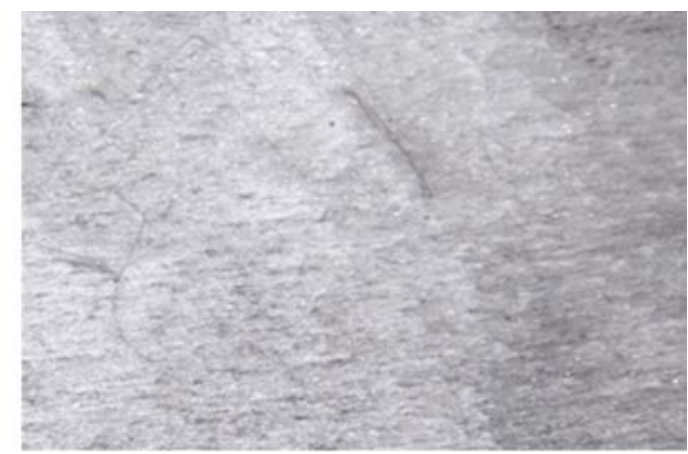

b)

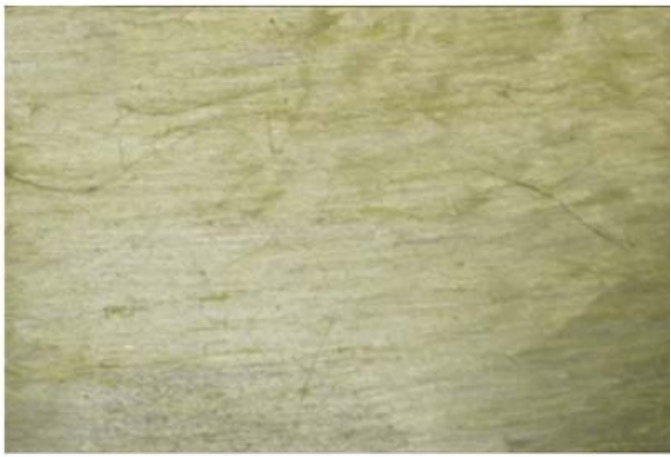

Figure 3. Photograph of (a) 100\% PCL (b) 100\% PVP (c) 50:50 PCL/PVP (d) drug loaded 50:50 PCL/PVP. The surface of $\mathrm{C}$-spun fibers are rough and lack luster where as with corporation of PVP, the samples look lustrous due to the presence of homogeneity in diameter of the fibers.

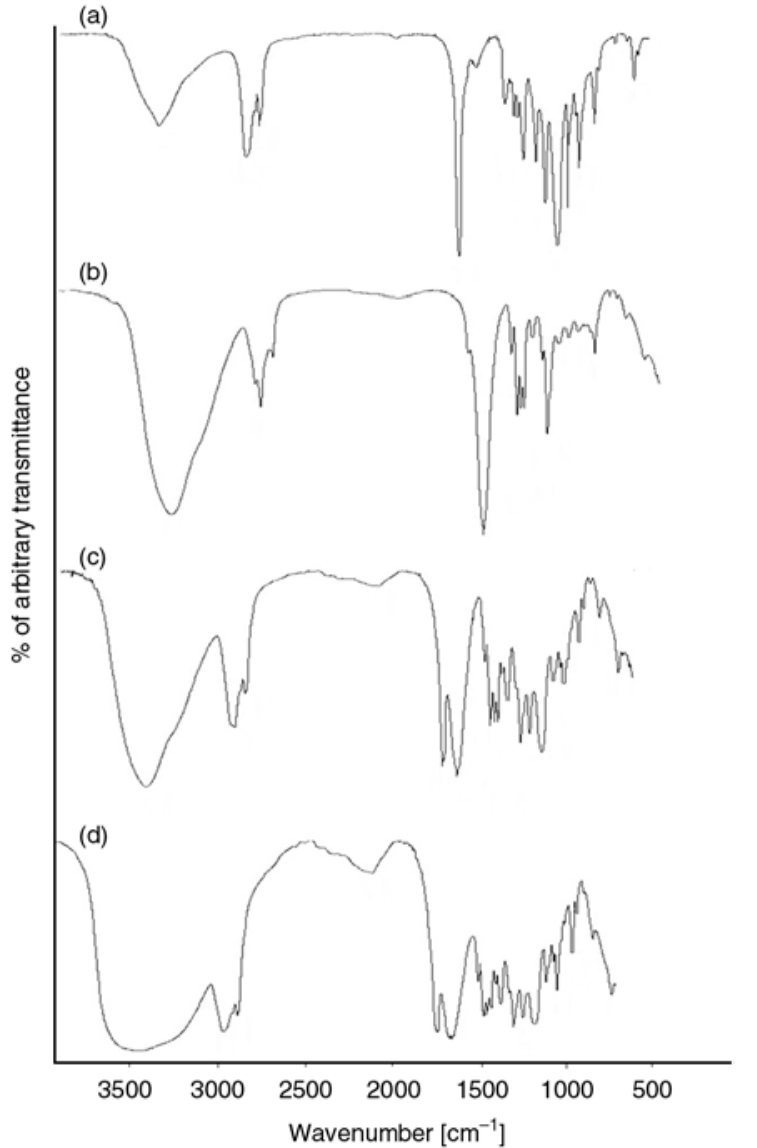

Figure 4. FTIR spectrum of (a) 100\% PCL (b) 100\% PVP (c) 50:50 PCL/PVP (d) drug loaded 50:50 PCL/ PVP)

\subsection{Thermo gravimetric analysis}

Thermal stability of polymers and their blends are evaluated by TGA. Various blend characteristics such as interaction and miscibility of the blends are evaluated by thermogram. The samples weighed $(\sim 10 \mathrm{mg})$ were heated from 0 to $700^{\circ} \mathrm{C}$ at $20^{\circ} \mathrm{C} / \mathrm{min}$ and held at an isotherm for $3 \mathrm{~min}$. The TGA data's were plotted as temperature verses weight $\%$, from which onset, peak and final decomposition temperature were obtained. Blending of Polymers results in negative (destabilizing) or positive (stabilizing) effect on thermal stability [26]. In Figure 5, neat PCL sample showed one step degradation profile as its degradation begins at $345^{\circ} \mathrm{C}$ and completely degraded around $490^{\circ} \mathrm{C}$. The similar results have been observed by Abdellatif Mohamed et al. [27] and proved that PCL showed much higher degradation temperature than vital gluten. In neat PVP samples initial weight loss nearly around 19\% below $100^{\circ} \mathrm{C}$ revealed the hydrophilic nature of PVP. PVP also shows one step degradation process and the degradation begins at $302^{\circ} \mathrm{C}$ and ends at $485^{\circ} \mathrm{C}$ as suggested in the literature [28]. Thermal behavior of PCL blended PVP obeys the single step degradation like PCL and PVP. The addition of tetracycline slightly improves the thermal stability of PCL 


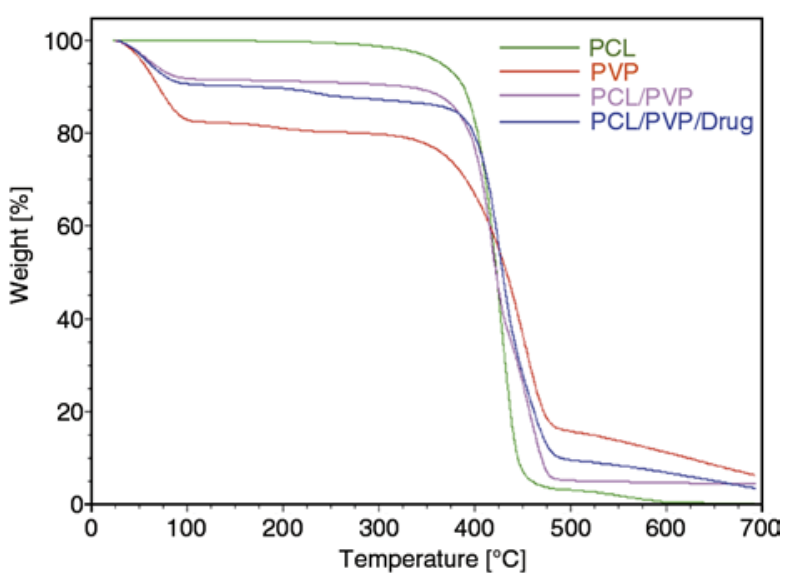

Figure 5. Thermogram of C-spun ultrafine fibrous web

blended PVP fibrous web and is evident from less residual weight obtained at elevated temperature. It also confirms that addition of drug has not significantly altered the crystallinity of the fibrous mat.

\subsection{Degree of swelling and weight loss [\%]}

Figures 6 and 7 shows the degree of swelling and weight loss [\%] of PCL, PVL/PVP, PVP ultrafine fibrous web kept in acetate buffer of $\mathrm{pH} 7.4$ at the intervals of 2, 4, 12 and 24 hours. Degree of swelling and weight loss [\%] are the two important parameters for designing drug delivery vehicle for particular biomedical application. From the Figure 6 it can be seen that PVP swells 583\% in first 2 hours and after two hours the entire fiber network collapses and starts slowly dissolving in the medium. It substantiates that a hydrophobic biocompatible polymer such as PCL along with the PVP will aid in stability of matrices so that the loaded drug release

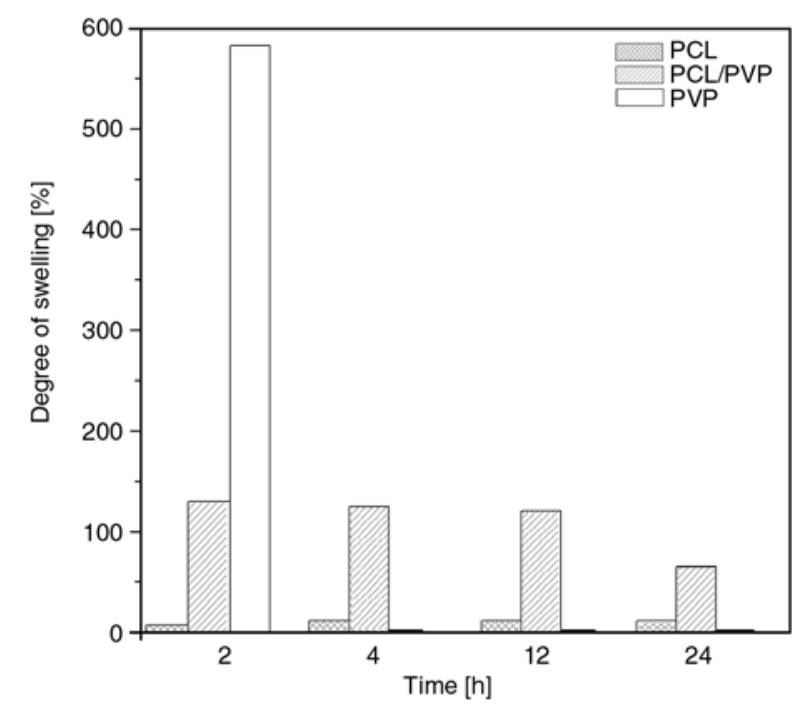

Figure 6. Degree of swelling for PCL, PCL/PVP, PVP ultrafine fibrous web

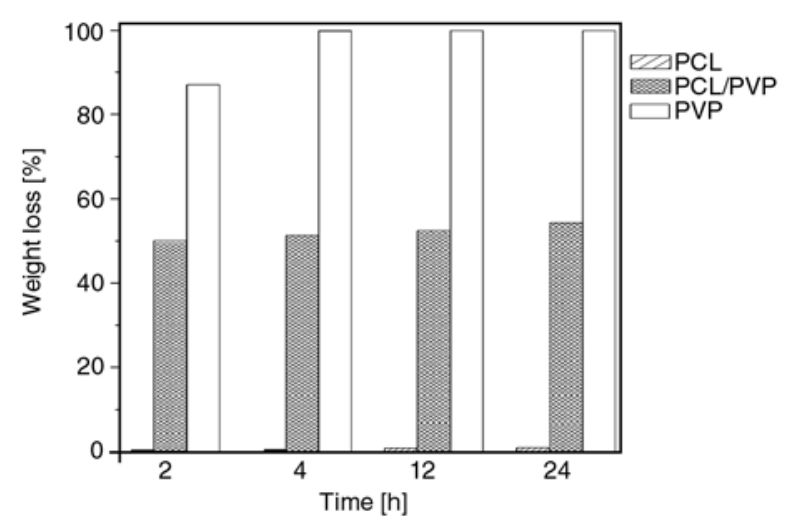

Figure 7. Weight loss [\%] for PCL, PCL/PVP, PVP ultrafine fibrous web

from the matrices can be maintained for longer duration. The degree of swelling of PCL/PVP blends was around $60-130 \%$ for time interval taken for the study whereas the hydrophobic PCL mat showed negligible swelling compared to the blends. The weight loss [\%] was found to be in the following order $P V P>P C L / P V P>P C L$ and PCL and PCL/ PVP blends were found to be more stable compared to the PVP matrices.

\subsection{Antibacterial activity}

The antibacterial efficiency of C-spun web was tested against two gram positive (S.epidermidis, B.megaterium) and gram negative bacteria (E.coli, P.aeruginosa) respectively. The microbes selected were skin pathogens and the developed C-spun fibrous web has a potential to be used as skin pathogens. The efficiency of the drug loaded C-spun fibres against skin pathogens is given in Table 2 and Figure 8 . It can be seen that the drug taken for the study namely tetracycline has a classic broad spectrum activity against both gram positive and gram negative bacterial infections. Tetracycline works by binding specifically to $30 \mathrm{~s}$ ribosomes of the bacteria, preventing attachment of aminoacyl tRNA to the tRNA - ribosome complex and simultaneously inhibits other steps of protein biosynthesis. Tetracycline released from the C-spun fibrous web effectively inhibits the growth of gram positive bacteria such as S.epidermidis and B.megaterium which gives the zone of inhibition $42 \mathrm{~mm} 46 \mathrm{~mm}$ and moderately inhibits the growth of gram negative bacterium such as E.coli and P.aeruginosa which gives the zone of inhibition around $33 \mathrm{~mm}, 31 \mathrm{~mm}$. The antimicrobial activity of tetracycline is more efficient to gram positive bacteria than gram nega- 


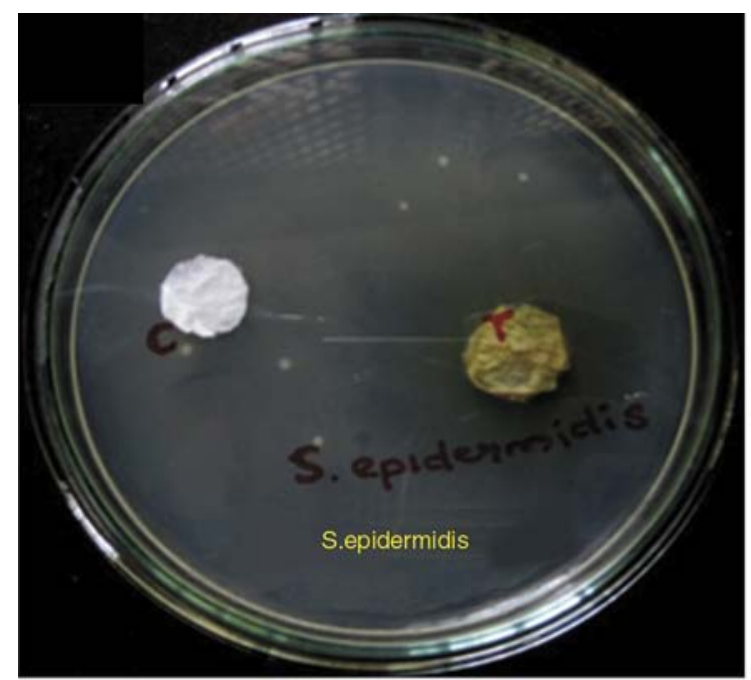

a)

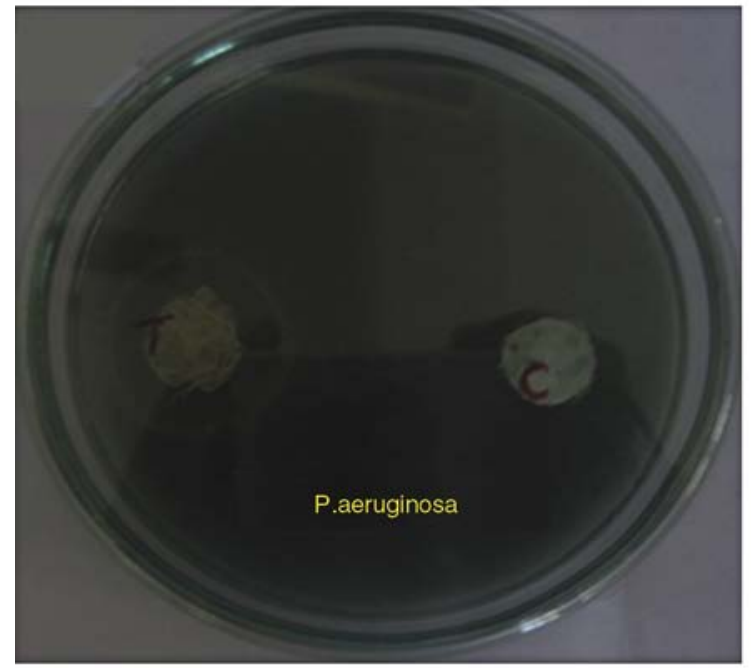

b)

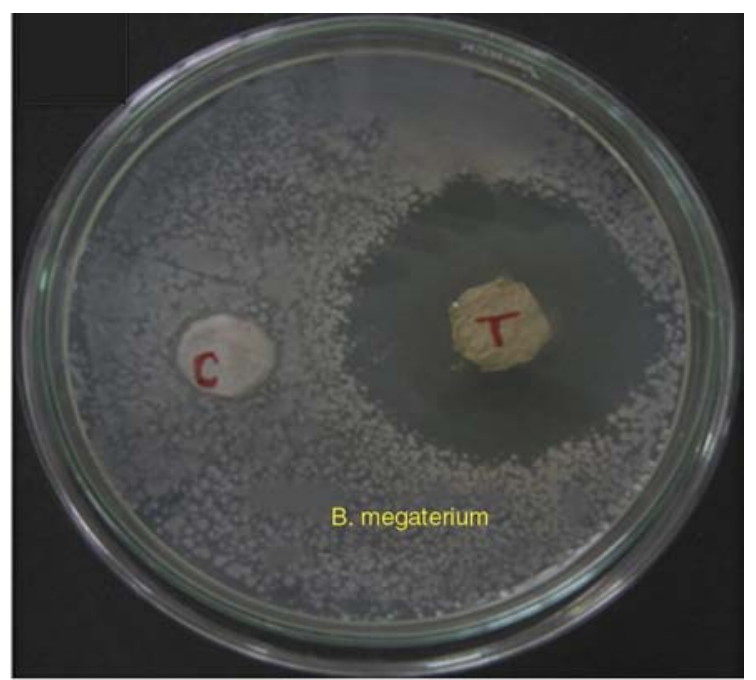

c)

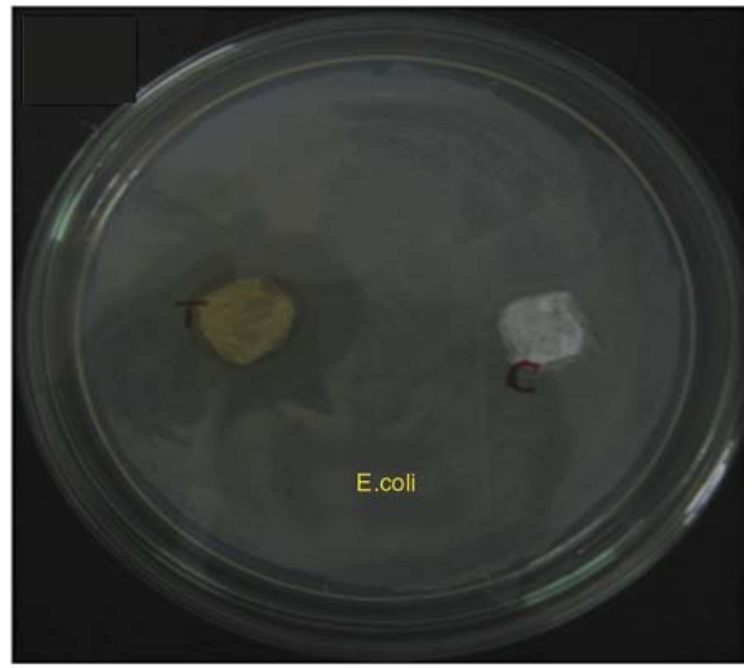

d)

Figure 8. Antibacterial activity of the C-spun fibrous web, (a) S.epidermidis, (b) P.aeruginosa, (c) B.megaterium, (d) E.coli

Table 2. Measurements of average zone of inhibition of C-spun ultrafine fibres

\begin{tabular}{|l|c|}
\hline \multicolumn{1}{|c|}{ Bacteria } & Zone of inhibition in $\mathbf{~ m m}$ \\
\hline S.epidermidis $(\mathrm{Gram}+\mathrm{ve})$ & 42 \\
\hline P.aeruginosa $(\mathrm{Gram}-\mathrm{ve})$ & 31 \\
\hline B.megaterium $(\mathrm{Gram}+\mathrm{ve})$ & 46 \\
\hline E.coli $($ Gram $-\mathrm{ve})$ & 33 \\
\hline
\end{tabular}

tive and it can be attribute the cell wall differentiations as reported in the literature [29].

\subsection{Drug release}

Figure 9 shows the release profile of tetracycline loaded C-spun fibers for both PCL, PCL/PVP blends. In the case of PCL polymer, the drug release is slow and only $12.5 \%$ of the drug is released within 24 hours even though PCL C-spun fibers provides better structural integrity. The slow release of the drug is attributed to the hydrophobic nature of PCL.

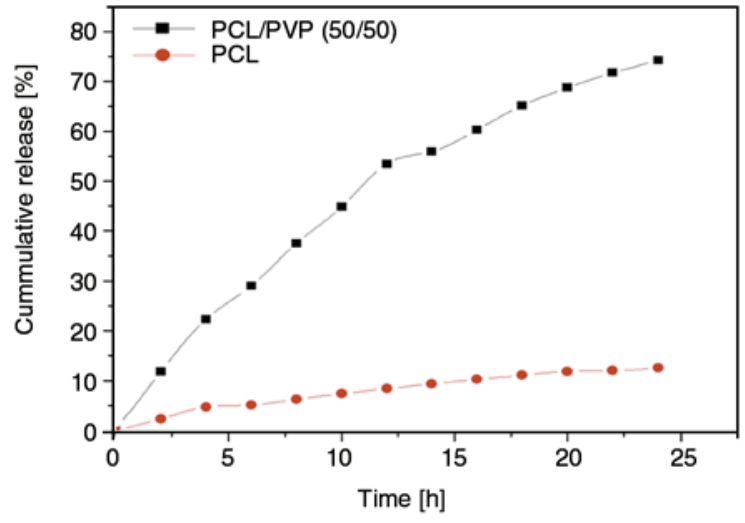

Figure 9. Drug releasing profiles of PCL/PVP C-spun ultrafine fibrous web

Blends of hydrophobic polymer along with hydrophilic polymer aids better drug release and it is evident from the drug release studies. It can be seen that around $74 \%$ of the drug is released within 24 hour in the case of PCL/PVP blends but still 


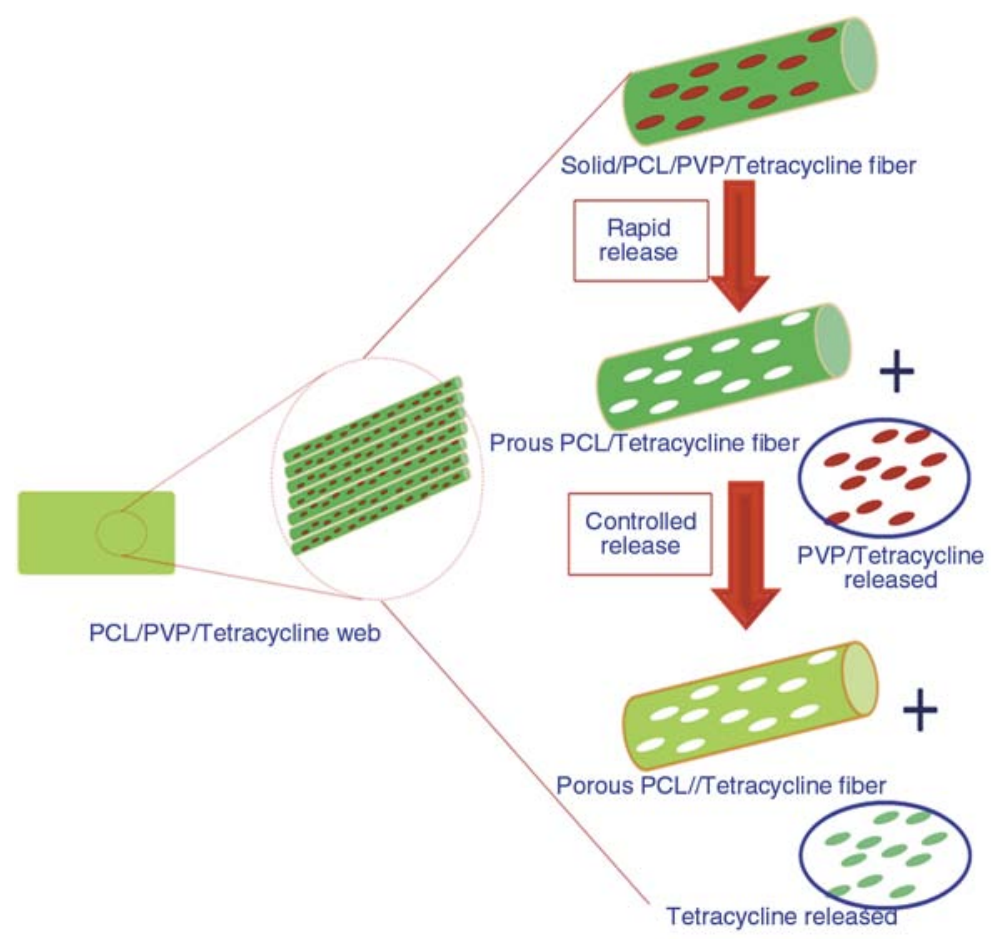

Figure 10. Schematic diagram of drug release from C-spun ultrafine fibrous web

drug is entrapped and released slowly. The conceptual diagram explaining the drug releasing mechanism is given in the Figure 10. From the figure it can be seen that drug loaded PCL/PVP fibers on contact with the releasing medium dissolves PVP readily and the drug which is intercalating with PVP is rapidly released leading to the formation of pores. The formed pores on C-spun matrices were confirmed by the SEM studies as shown in the Figure 11. Pores are uniformly distributed over the surface and the average pore size is found to be $375 \mathrm{~nm}$. The developed drug loaded ultrafine fibrous web can act as a perfect drug releasing vehicle where the rapid release of the drug needed to inhibit the bacteria causing dermal infections followed by sustained release to prevent secondary infections. The addition of hydrophilic polymer has a unique advantage of the fibrous web as it aids cell adhesion and proliferation thereby acting as good wound dressing material to inhibit the microorganism [30]. Moreover the release profiles were analyzed by various models Zero order, First order, Higuchi, Hixson-crowell and Peppas model. The results of the peppas model and others are tabulated in Table 3 and Table 4 . The co-efficient of determination values provide an indicator of how well the model can explain the variations is given in both the tables. From the tables it can be seen that the co-efficient of determination value of 0.99 is obtained for the

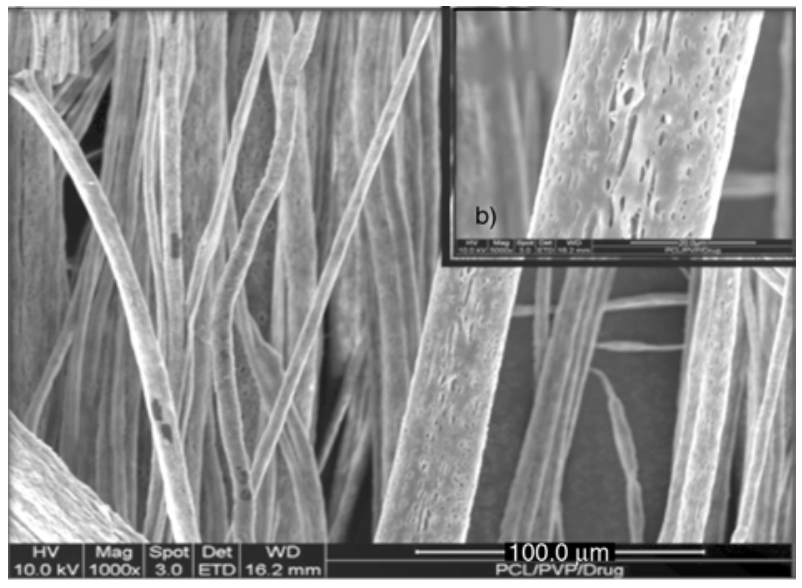

a)

Figure 11. SEM images after drug release, (a) fibrous web, (b) single fibre. Drug loaded PCL/PVP fibres on contact with the releasing medium dissolves PVP readily and the drug which is intercalating with PVP is rapidly released leading to the formation of pores.

Table 3. Diffusion constant $(n)$ and regression coefficient of Tetracycline release from PCL, PCL/PVP blend ultrafine fibre mats calculated by Peppas equation

\begin{tabular}{|l|c|c|}
\hline \multicolumn{1}{|c|}{ Ultra fine fiber type } & $\mathbf{r}^{\mathbf{2}}$ & $\mathbf{n}$ \\
\hline PCL & 0.99 & 0.628 \\
\hline PCL/PVP & 0.98 & 0.631 \\
\hline
\end{tabular}

Peppas model for both PVP and PCL/PVP indicating that the model best suits the release profile of the mat taken for the study. The $n$ values in the 
Table 4. Regression coefficients of different mathematical models fitted to the release of Tetracycline from PCL/PVP blend ultrafine fiber mats

\begin{tabular}{|l|c|c|c|c|}
\hline \multicolumn{1}{|c|}{ Ultra fine fiber type } & $\begin{array}{c}\text { Zero order } \\
\left(\mathbf{r}^{\mathbf{2}}\right)\end{array}$ & $\begin{array}{c}\text { First order } \\
\left(\mathbf{r}^{\mathbf{2}}\right)\end{array}$ & $\begin{array}{c}\text { Higuchi model } \\
\left(\mathbf{r}^{\mathbf{2}}\right)\end{array}$ & $\begin{array}{c}\text { Hixon- crowell Model } \\
\left(\mathbf{r}^{\mathbf{2}}\right)\end{array}$ \\
\hline PCL & 0.82 & 0.86 & 0.96 & 0.84 \\
\hline PCL/PVP & 0.81 & 0.98 & 0.94 & 0.958 \\
\hline
\end{tabular}

range of 0.628 suggest that the release behavior is both diffusion and swelling controlled drug release. The Higchui model also closely fits to the experimental data with $r^{2}$ of 0.96 and 0.94 for the PCL and PCL/PVP matrices respectively, suggesting the predominance of diffusion controlled release in the mat as suggested in literature.

\section{Conclusions}

Tetracycline incorporated PCL/PVP blended ultrafine fibrous web has been successively developed by our in-house built centrifugal spinning system. The developed fibrous web proved rapid drug release followed by sustained release and good antimicrobial activity against the tested pathogenic bacteria which is commonly found in dermal infections. The observed results concluded that it is possible to develop PCL/PVP blended ultrafine fibrous web by centrifugal spinning system and the developed web can be effectively used a wound dressing applications.

\section{Acknowledgements}

The Authors L. Amalorpava Mary \& V. R. Giridev would like to acknowledge Indian Council of Medical Research (ICMR) for funding (Proceeding No: 5/20-6(Bio)/11-NCDI). One of the authors wish to thank L. Sathish Kumar for helping to assemble the centrifugal spinning system.

\section{References}

[1] Zahedi P., Rezaeian I., Siadat S. O., Jafari S. H., Supaphol P.: A review on wound dressings with an emphasis on electrospun nanofibrous polymeric bandages. Polymers for Advanced Technologies, 21, 21-77 (2010). DOI: $10.1002 /$ pat.1625

[2] Hong K. H., Park J. L., Sul I. H., Youk J. H., Kang T. J.: Preparation of antimicrobial poly(vinyl alcohol) nanofibers containing silver nanoparticles. Journal of Polymer Science Part B: Polymer Physics, 44, 24682474 (2006).

DOI: $10.1002 /$ polb.20913
[3] Puppi D., Piras A. M., Detta N., Dinucci D., Chiellini F.: Poly(lactic-co-glycolic acid) electrospun fibrous meshes for the controlled release of retinoic acid. Acta Biomaterialia, 6, 1258-1268 (2010).

DOI: $10.1016 /$ j.actbio.2009.08.015

[4] Ma P. X., Zhang R.: Synthetic nano-scale fibrous extracellular matrix. Journal of Biomedical Materials Research, 46, 60-72 (1999).

DOI: 10.1002/(SICI)1097-4636(199907)46:1<60::AIDJBM7>3.0.CO;2-H

[5] Yang F., Murugan R., Ramakrishna S., Wang X., Ma Y-X., Wang S.: Fabrication of nano-structured porous PLLA scaffold intended for nerve tissue engineering. Biomaterials, 25, 1891-1900 (2004).

DOI: $10.1016 /$ j.biomaterials.2003.08.062

[6] Senthilram T., Mary L. A., Venugopal J. R., Nagarajan L., Ramakrishna S., Dev V. R. G.: Self crimped and aligned fibers. Materials Today, 14, 226-229 (2011). DOI: $10.1016 / \mathrm{S} 1369-7021(11) 70118-3$

[7] Lin S-Y., Chen K-S., Run-Chu L.: Design and evaluation of drug-loaded wound dressing having thermoresponsive, adhesive, absorptive and easy peeling properties. Biomaterials, 22, 2999-3004 (2001).

DOI: 10.1016/S0142-9612(01)00046-1

[8] Purna S. K., Babu M.: Collagen based dressings - A review. Burns, 26, 54-62 (2000).

[9] William H., Eaglstein M. D.: Moist wound healing with occlusive dressings: A clinical focus. Dermatologic Surgery, 27, 175-182 (2001).

DOI: 10.1046/j.1524-4725.2001.00299.x

[10] Pitt C. G.: Poly ( $\varepsilon$-caprolactone) and its co-polymers. in 'Biodegradable polymers as drug delivery systems' (eds.: Chasin M., Langer R.) Marcel Decker, New York, 71-120 (1990).

[11] Luong-Van E., Grøndahl L., Chua K. N., Leong K. W., Nurcombe V., Cool S. M.: Controlled release of heparin from poly( $\varepsilon$-caprolactone) electrospun fibers. Biomaterials, 27, 2042-2050 (2006).

DOI: $10.1016 /$ j.biomaterials.2005.10.028

[12] Heunis T. D. J., Dicks L. M. T.: Nanofibers offer alternativeways to the treatment of skin infections. Journal of Biomedicine and Biotechnology, 2010, 510682/1510682/10 (2010).

DOI: $10.1155 / 2010 / 510682$

[13] Yang Q., Li Z., Hong Y., Zhao Y., Qiu S., Wang C., Wei Y.: Influence of solvents on the formation of ultrathin uniform poly(vinyl pyrrolidone) nanofibers with electrospinning. Journal of Polymer Science Part B: Polymer Physics, 42, 3721-3726 (2004),

DOI: $10.1002 /$ polb.20222 
[14] Zamani M., Morshed M., Varshosaz J., Jannesari M.: Controlled release of metronidazole benzoate from poly $\varepsilon$-caprolactone electrospun nanofibers for periodontal diseases. European Journal of Pharmaceutics and Biopharmaceutics, 75, 179-185 (2010). DOI: $10.1016 /$ j.ejpb.2010.02.002

[15] Kanawung K., Panitchanapan K., Puangmalee S-O., Utok W., Kreua-ongarjnukool N., Rangkupan R., Meechaisue C., Supaphol P.: Preparation and characterization of polycaprolactone/diclofenac sodium and poly(vinyl alcohol)/tetracycline hydrochloride fiber mats and their release of the model drugs. Polymer Journal, 39, 369-378 (2007).

DOI: $10.1295 /$ polymj.PJ2006011

[16] Kenawy E-R., Bowlin G. L., Mansfield K., Layman J., Simpson D. G., Sanders E. H., Wnek G. E.: Release of tetracycline hydrochloride from electrospun poly(ethylene-co-vinylacetate), poly(lactic acid), and a blend. Journal of Controlled Release, 81, 57-64 (2002).

DOI: $10.1016 / \mathrm{S} 0168-3659(02) 00041-\mathrm{X}$

[17] Taepaiboon P., Rungsardthong U., Supaphol P.: Drugloaded electrospun mats of poly(vinyl alcohol) fibres and their release characteristics of four model drugs. Nanotechnology, 17, 2317-2329 (2006).

DOI: $10.1088 / 0957-4484 / 17 / 9 / 041$

[18] Jannesari M., Varshosaz J., Morshed M., Zamani M.: Composite poly(vinyl alcohol)/poly(vinyl acetate) electrospun nanofibrous mats as a novel wound dressing matrix for controlled release of drugs. International Journal of Nanomedicine, 6, 993-1003 (2011). DOI: $10.2147 /$ IJN.S17595

[19] Varelas C. G., Dixon D. G., Steiner C. A.: Zero-order release from biphasic polymer hydrogels. Journal of Controlled Release, 34, 185-192 (1995). DOI: 10.1016/0168-3659(94)00085-9

[20] Mulye N. V., Turco S. J.: A simple model based on first order kinetics to explain release of highly water soluble drugs from porous dicalcium phosphate dihydrate matrices. Drug Development and Industrial Pharmacy, 21, 943-953 (1995). DOI: $10.3109 / 03639049509026658$

[21] Higuchi T.: Rate of release of medicaments from ointment bases containing drugs in suspension. Journal of Pharmaceutical Sciences, 50, 874-875 (1961). DOI: $10.1002 / j p s .2600501018$

[22] Niebergall P. J., Milosovich G., Goyan J. E.: Dissolution rate studies II. Dissolution of particles under conditions of rapid agitation. Journal of Pharmaceutical Sciences, 52, 236-241 (1963). DOI: $10.1002 / j p s .2600520310$
[23] Peppas N. A.: Analysis of Fickian and non-Fickian drug release from polymers. Pharmaceutica Acta Helvitiae, 60, 110-111 (1985).

[24] Meng Z. X., Zheng W., Li L., Zheng Y. F.: Fabrication, characterization and in vitro drug release behavior of electrospun PLGA/chitosan nanofibrous scaffold. Materials Chemistry and Physics, 125, 606-611 (2011). DOI: 10.1016/j.matchemphys.2010.10.010

[25] Zhong S., Teo W. E., Zhu X., Beuerman R. W., Ramakrishna S., Yung L. Y. L.: An aligned nanofibrous collagen scaffold by electrospinning and its effects on in vitro fibroblast culture. Journal of Biomedical Materials Research Part A, 79, 456-463 (2006).

DOI: 10.1002/jbm.a.30870

[26] Biresaw G., Mohamed A., Gordon S. H., HarryO'kuru R. E., Carriere C. C.: Compatibility study in poly (tetramethyleneadipate-co-terephthalate)/polystyrene bioblends. Journal of Applied Polymer Science, 110, 2932-2941 (2008).

DOI: 10.1002/app.28893

[27] Mohamed A., Finkenstadt V. L., Gordon S. H., Biresaw G., Palmquist D. E., Rayas-Duarte P.: Thermal properties of PCL/gluten bioblends characterized by TGA, DSC, SEM, and infrared-PAS. Journal of Applied Polymer Science, 110, 3256-3266 (2008).

DOI: 10.1002/app.28914

[28] Moharram M. A., Khafagi M. G.: Thermal behavior of poly(acrylic acid)-poly(vinyl pyrrolidone) and poly (acrylic acid)-metal-poly(vinyl pyrrolidone) complexes. Journal of Applied Polymer Science, 102, 4049-4057 (2006).

DOI: $10.1002 / a p p .24367$

[29] Zahedi P., Karami Z., Rezaeian I., Jafari S-H., Mahdaviani P., Abdolghaffari A. H., Abdollahi M.: Preparation and performance evaluation of tetracycline hydrochloride loaded wound dressing mats based on electrospun nanofibrous poly(lactic acid)/poly(e-caprolactone) blends. Journal of Applied Polymer Science, 124, 4174-4183 (2012).

DOI: $10.1002 / a p p .35372$

[30] Wan Y., Chen W., Yang J., Bei J., Wang S.: Biodegradable poly(L-lactide)-poly(ethylene glycol) multiblock copolymer: Synthesis and evaluation of cell affinity. Biomaterials, 24, 2195-2203 (2003). DOI: $10.1016 / \mathrm{S} 0142-9612(03) 00107-8$ 